\title{
Using Flow Feature to Extract Pulsatile Blood Flow from 4D Flow MRI Images
}

\author{
Zhiqiang Wang ${ }^{\mathrm{a}}$, Ye Zhao ${ }^{\mathrm{a}}$, Whitney $\mathrm{Yu}^{\mathrm{b}}$, Xi Chen ${ }^{\mathrm{b}}$, Chen Lin $^{\mathrm{c}}$, Stephen F Kralik ${ }^{\mathrm{c}}$, and Gary \\ D Hutchins ${ }^{\mathrm{c}}$ \\ ${ }^{a}$ Computer Science Department, Kent State University, Kent, USA \\ ${ }^{\mathrm{b}}$ Mechanical Engineering Department, Indiana University-Purdue University, Indianapolis, \\ USA \\ ${ }^{c}$ Radiology and Imaging Sciences Department, Indiana University-Purdue University, \\ Indianapolis, USA
}

\begin{abstract}
4D flow MRI images make it possible to measure pulsatile blood flow inside deforming vessel, which is critical in accurate blood flow visualization, simulation, and evaluation. Such data has great potential to overcome problems in existing work, which usually does not reflect the dynamic nature of elastic vessels and blood flows in cardiac cycles. However, the 4D flow MRI data is often low-resolution and with strong noise. Due to these challenges, few efforts have been successfully conducted to extract dynamic blood flow fields and deforming artery over cardiac cycles, especially for small artery like carotid. In this paper, a robust flow feature, particularly the mean flow intensity is used to segment blood flow regions inside vessels from 4D flow MRI images in whole cardiac cycle. To estimate this flow feature more accurately, adaptive weights are added to the raw velocity vectors based on the noise strength of MRI imaging. Then, based on this feature, target arteries are tracked in at different time steps in a cardiac cycle. This method is applied to the clinical 4D flow MRI data in neck area. Dynamic vessel walls and blood flows are effectively generated in a cardiac cycle in the relatively small carotid arteries. Good image segmentation results on 2D slices are presented, together with the visualization of 3D arteries and blood flows. Evaluation of the method was performed by clinical doctors and by checking flow volume rates in the vertebral and carotid arteries.
\end{abstract}

Keywords: 4D Flow MRI, Image Segmentation, flow feature, Mean Flow Intensity

\section{INTRODUCTION}

Recently, time-resolved Phase Contrast Magnetic Resonance Imaging with 3D velocity encoding of blood flow (also referred as 4D flow MRI) has been developed as a versatile and non-invasive tool for cardiovascular flow assessment. 4D flow MRI data provides information about the spatial and temporal evolution of 3D blood flow within vascular region of interest. Thus, it can help clinicians and researchers to comprehensively evaluate blood flow patterns by visualizing and quantifying 3D blood flows on flow volume, pressure change, Wall Shear Stress (WSS), turbulence intensity, and many other useful hemodynamic properties. ${ }^{1}$ Extracting accurate blood flows is a key step for post-processing tasks such as visualization and quantitative studies. Most existing approaches ${ }^{2-4}$ extract peak systole blood velocities, when the flow velocity is the highest during one cardiac cycle. The movement of the elastic vessels over cardiac cycles is not considered which may lead to inaccurate flows. Consequently, the computation of critical flow attributes becomes less accurate, such as the wall shear stress closely associated with atherosclerosis. ${ }^{5}$ Therefore, it is preferable to extract dynamic pulsatile blood flows from 4D flow MRI data. Few existing work has been conducted in this direction. A recent work ${ }^{6}$ uses the magnitude of velocities to segment thoracic vessels in a cardiac cycle. However, the magnitudes change largely in a cycle. For smaller arteries, the magnitudes in diastole become rather small making it difficult to distinguish blood flow field from background.

Contact author: Zhiqiang Wang

E-mail: zwang22@kent.edu

Medical Imaging 2017: Image Processing, edited by Martin A. Styner, Elsa D. Angelini, Proc. of SPIE Vol. 10133, $1013310 \cdot$ C 2017 SPIE · CCC code: 1605-7422/17/\$18 · doi: 10.1117/12.2249500 
The velocity-valued images from 4D flow MRI scan is a typical multiple channel image dataset like color image. In color image processing, vector based techniques which treat multiple channel information in its entirety as vectors often yields more high-quality and robust results, ${ }^{7-9}$ since they consider the relationship among vector components. However, these methods mostly use the image features, such as intensity gradient, texture etc., in the processing work like segmentation. One the other hand, Particle Image Velocimetry (PIV) as optical measurement method, its images can also record dynamic flow velocity information. With high resolution camera and laser illumination, PIV as an invasive method can achieve high spatial resolution with less noise. ${ }^{10}$ Comparing to PIV, noninvasive methods such as 4D flow MRI data usually has less image contrast and strong noise, and records limited spatial information. Thus, design image processing algorithms over 4D flow MRI data is an new challenge task.

Considering 4D flow MRI's measurement result is actually physical blood flows, the flow field should meet flow dynamic characters. In this work, a flow feature, the mean flow intensity is used, which provides a more reliable metric to distinguish blood flow from background in a whole cardiac cycle. And then, the extracted dynamic boundaries are used to define deformation factors at different spatial locations of the vessel and at different time steps in cardiac cycles. These factors enable the generation of high quality and deforming vessel walls, by morphing the wall acquired from accurate but static TOF (Time of Fight) MRI anatomic images. The deforming walls facilitate the extraction of pulsatile blood flow directly, so that they can be used together in blood flow and vessel visualization and assessment.

In the following, we first show how the fluid feature can be used to extract blood flow region in Sec. 2 . Based on the extracted flow field, we describe the method for generating pulsatile vessel boundary for flow field visualization in Sec. 3. Then, we tested this method for vertebral and carotid vessels which is discussed in Sec. 4 and evaluated it at Sec. 5 .

\section{FLOW FIELD EXTRACTION BASED ON MEAN FLOW INTENSITY}

In particular, we compute a mean flow intensity on each image slices, which can robustly distinguish velocities from background noise in different time steps of a cardiac cycle. In the computation, we also add adaptive weights to raw velocity corresponding to its estimated noise strength. Then, using active contour model with mean flow intensity to extract flow field. The feature model is analogous to the phase congruency method in image processing, which is known to be robust over image illumination variations. Therefore, the approach is good at segmenting arteries having a large difference of blood velocity magnitude between systole and diastole.

\subsection{Mean Flow Intensity}

The critical point of effective segmentation for $4 \mathrm{D}$ flow MRI images is to find a robust feature that can reflect blood flow field in a whole cardiac cycle. Some fluid mechanical attributes such as divergence and vorticity involving differential operators are sensitive to outliers and noise in raw vector data. Meanwhile the fluid attributes utilizing local aggregations such as mean, variance, degree of turbulence were less severely affected by noise data. ${ }^{11}$ Among these attributes, we found the mean flow intensity has the optimal performance in velocity field extraction.

Through a Reynolds decomposition, ${ }^{12}$ an instantaneous flow velocity $\vec{U}$ can be divided to two components: mean flow part $\overrightarrow{U_{m}}$ and turbulence flow part $\overrightarrow{U_{r}}$ as:

$$
\vec{U}=\overrightarrow{U_{m}}+\overrightarrow{U_{r}}
$$

And the mean flow intensity $I_{m}$ is set as:

$$
I_{m}=\frac{\left\|\overrightarrow{U_{m}}\right\|}{\|\vec{U}\|}
$$

which is the strength of mean flow over the local flow strength. Since the vectors representing background noise in the 4D flow MRI data is randomly distributed in spatial and temporal domain, their fluctuation is very large (in some sense, extremely turbulent) and the strength of mean flow is close to 0 , which would yield very small $I_{m}$. In contrast, blood transportation in arteries is usually viscous and laminar flow especially close to the boundary, 
where velocity vectors are strongly oriented. It would yield large mean flow strength and rather high $I_{m}$. Thus mean flow intensity can be used to characterize stable flow behavior in blood flows and as an indicator to differ from the background area.

To calculate $I_{m}$, the mean flow velocity $\overrightarrow{U_{m}}$ can be estimated through a $4 \mathrm{D}$ Gaussian kernel in spatial and temporal domain: ${ }^{13}$

$$
\overrightarrow{U_{m}}(x, t)=\sum_{\left(x_{i}, t_{j}\right) \in \Omega} \vec{U}\left(x_{i}, t_{j}\right) g_{s}\left(\left\|x_{i}-x\right\|\right) g_{t}\left(\left\|t_{i}-t\right\|\right)
$$

where $\vec{U}(x, t)$ is the original velocity vector at location $x$ and time step $t$ in $4 \mathrm{D}$ domain, $\Omega$ is a $4 \mathrm{D}$ window centered in $(x, t)$, and $\vec{U}\left(x_{i}, t_{j}\right)$ is a neighbor vector in this window. $g_{s}$ and $g_{t}$ are the Gaussian kernels with variance $s$ and $t$ in spatial and temporal domain, respectively. For simplicity, in the following, $g_{s}\left(\left\|x_{i}-x\right\|\right) g_{t}\left(\left\|t_{i}-t\right\|\right)$ is termed as the $4 \mathrm{D}$ kernel $w\left(x_{i}, t_{j}\right)$. The local flow strength $\|\vec{U}\|$ can also be estimated as

$$
\|\vec{U}(x, t)\|_{E}=\sum_{\left(x_{i}, t_{j}\right) \in \Omega}\|\vec{U}(x, t)\| w\left(x_{i}, t_{j}\right)
$$

Therefore, Based on Equation 2, Equation 3 and Equation 4, $I_{m}$ is calculated as:

$$
I_{m}(x, t)=\frac{\left\|\overrightarrow{U_{m}}(x, t)\right\|}{\left\|\vec{U}\left(x_{i}, t_{j}\right)\right\|_{E}}=\frac{\left\|\sum_{\left(x_{i}, t_{j}\right) \in \Omega} \vec{U}\left(x_{i}, t_{j}\right) w\left(x_{i}, t_{j}\right)\right\|}{\sum_{\left(x_{i}, t_{j}\right) \in \Omega}\left\|\vec{U}\left(x_{i}, t_{j}\right)\right\| w\left(x_{i}, t_{j}\right)}
$$

Since $0 \leq\left\|\sum_{\left(x_{i}, t_{j}\right) \in \Omega} \vec{U}(x, t) w\left(x_{i}, t_{j}\right)\right\| \leq \sum_{\left(x_{i}, t_{j}\right) \in \Omega}\left\|\vec{U}\left(x_{i}, t_{j}\right)\right\| w\left(x_{i}, t_{j}\right)$, the range of $I_{m}(x, t)$ is $0 \leq I_{m}(x, t) \leq 1$.

\subsection{Weighting Velocity Vector based on Imaging Quality}

To estimate $I_{m}(x, t)$ more accurately, we add weights to the raw velocity vectors based on the specific sampling quality of MRI imaging. The quality is measured by a strength level of noise $\sigma_{v}:{ }^{5}$

$$
\sigma_{v}=\frac{\pi V_{e n c}}{\sqrt{2} S N R_{M}}
$$

Where $V_{\text {enc }}$ is set before MRI scanning representing the maximum flow velocity that can be measured without alias in each velocity channel, which is set by MRI operators before scanning. In practice, the selected $V_{\text {enc }}$ is varied set for each channel according to the flow velocity range in each direction to suppress noise. Where $S N R_{M}$ is the Signal Noise Ratio of the corresponding MRI magnitude image M. Noise in magnitude image M is usually modeled by Rician distribution, which is signal dependent and its mean value depends on local intensity $M(x, t)$ in the magnitude image. The $S N R_{M}$ can be estimated locally for each location. ${ }^{14}$ Therefore, before computing $I_{m}(x, t)$, the raw velocities $\vec{U}$ in three direction are weighted inversely to the noise strength $\sigma_{v}$ as:

$$
U_{x}^{\prime}=U_{x} \frac{S N R_{M}}{V_{\text {enc_ } x}}, U_{y}^{\prime}=U_{y} \frac{S N R_{M}}{V_{\text {enc_y }}}, U_{z}^{\prime}=U_{z} \frac{S N R_{M}}{V_{\text {enc_z }}} .
$$

Where $V_{\text {enc } \_x}, V_{\text {enc_y } y}, V_{\text {enc } z}$ are $V_{\text {enc }}$ in the 3 velocity channels.

\subsection{Feature Robustness and Computing Performance}

To show this feature is robust to the dynamic change of velocity field in the whole cardiac cycle, here we show how this is achieved by linking this method to phase congruency. It is known that phase congruency is an image feature detection method in frequency domain, which is particularly robust against changes in image illumination. ${ }^{15}$

Our model can be considered as a weighted phase congruency estimator for velocity vectors in spatial and temporal domain. If consider the weighted velocity vector as $\overrightarrow{U^{\prime \prime}}$, Assuming $\overrightarrow{U^{\prime \prime}}\left(x_{i}, t_{j}\right)$ has its magnitude as $A\left(x_{i}, t_{j}\right)$ and its phase as $\phi\left(x_{i}, t_{j}\right)$. Then the sum of these vectors, named $N A_{a}$, is a vector. Fig. 1a explains this computation in vector geometry. A sequence of velocities $\overrightarrow{U^{\prime}}\left(x_{i}, t_{j}\right)$ are added consecutively, where each vector 


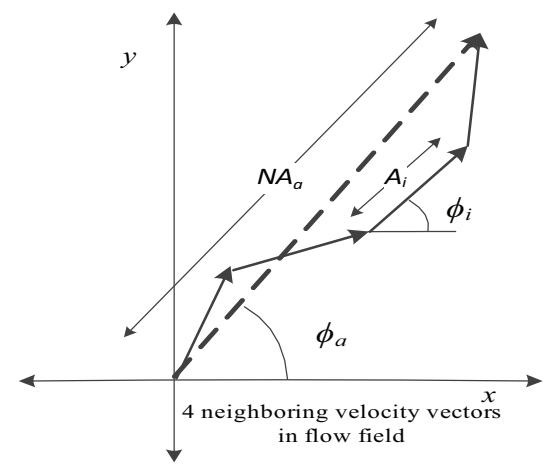

(a) Blood flow field

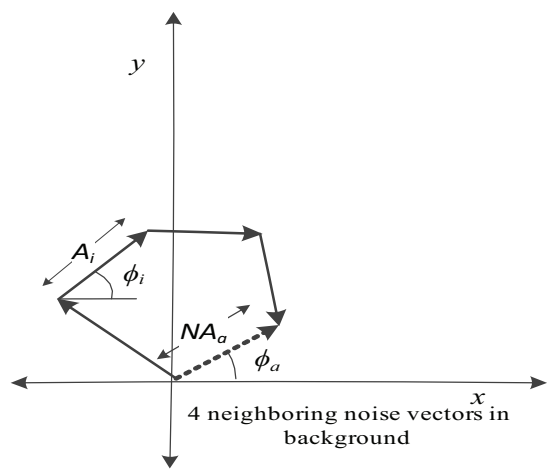

(b) Noise field

Figure 1. Phase congruency of 4 neighboring vectors: (a) in flow field; (b) in background noise field. The vectors are plotted head to tail. Neighboring velocity vectors in flow field have high phase coherence and yield high phase congruency value. In contrast, noise vectors are randomly distributed making value of phase congruency very small.

is drawn as a vector with length $A_{i}$, and phase angle $\phi_{i}$. The result vector $N A_{a}$ has the angle $\phi_{a}$. Based on Equation 5 , the mean velocity intensity can also be calculated as:

$$
I_{m}(x, t)=\frac{\sum_{\left(x_{i}, t_{j}\right) \in \Omega} A\left(x_{i}, t_{j}\right) \cos \left(\phi\left(x_{i}, t_{j}\right)-\phi_{a}(x, t)\right)}{\sum_{\left(x_{i}, t_{j}\right) \in \Omega} A\left(x_{i}, t_{j}\right)} .
$$

Here, the addition of phase angles achieves phase congruency value accumulated from every neighbor vector orientation in the local window $\Omega$.

For blood flow field, the vector phase difference between the neighboring velocity vectors is relative small. So length of $N A_{a}$ is rather large and the phase congruency value close to 1 . On the other hand, since there is no coherence of phase angles in the background noise field. As shown in Figure 1b, the value would fall to a minimum close to 0 . Thus, the computation model of $I_{m}(x, t)$ can be considered as a phase congruency estimator in spatial and temporal domain. This measure is independent on overall velocity magnitude, so it is very robust to velocity magnitude variations of pulsatile blood flow in different time steps. Therefore, even using a fixed threshold can lead to good segmentation results for all images in a whole cardiac cycle.

For Computational efficiency in Equation 5, it only needs to traverse an image once. The 4D Gaussian convolution operations can be computed fast by implementing $1 \mathrm{D}$ convolution kernel separately on each dimension, ${ }^{16}$ which has been widely used in image bilateral filter methods. As a result, a desktop with a consumer CPU can compute mean flow intensity map of hundreds images in several seconds.

\subsection{Blood Flow Extraction based on Mean Flow Intensity}

Based on $I_{m}(x, t)$, each slice of velocity images becomes an intensity image. It can be easily segmented through one uniform threshold $\gamma$ to segment flow field regions from the background. The threshold can be estimated according to the static features of the image. For instance, we can use a small area on an image corner to fit a normal distribution with mean value $\mu$ and variance $\sigma$, then this threshold is set to $\mu+2 \sigma$. These parameters can be adjusted after processing a small data set, then apply them to large data set automatically. To automatically extract target flow field in 4D space and keep boundary smooth, an active contour model with level set representation is set as:

$$
\frac{\partial \psi}{\partial t}=\operatorname{div}\left(\lambda \frac{\nabla \psi}{|\nabla \psi|}\right)+\left(I_{m}-\gamma\right)|\nabla \psi|
$$

Where $\psi$ is the signed distance field, the first term on the right is a smooth term with weight $\lambda$, and the second term drives the contour towards a given threshold $\gamma$. 


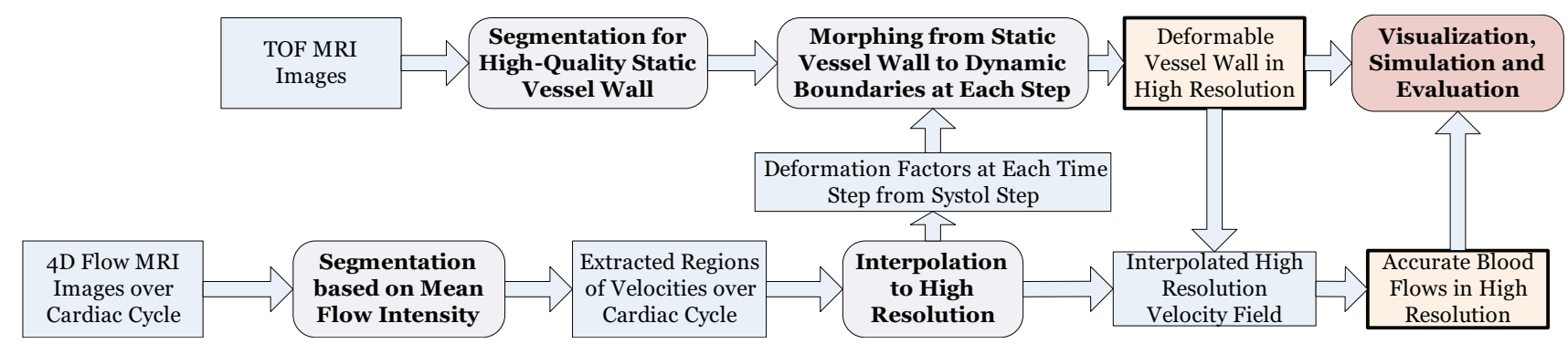

Figure 2. Generate High Quality Deforming Vessel Boundary for Flow Field Visualization

\section{GENERATE DEFORMING VESSEL BOUNDARY FOR FLOW VISUALIZATION}

After flow region is extracted over each MRI slice, applying the method over different images sampled along cardiac time steps $t$, the dynamic vessel walls $\Lambda_{t}$, can be extracted and reconstructed. At the same time, the velocity fields $\vec{U}_{t}$ inside these regions are extracted to get pulsatile blood flow varying over $t$. However, these results suffer from the low-resolution problem. For example, a carotid vessel is scanned in a resolution about $0.4 \mathrm{~mm} \times 0.4 \mathrm{~mm} \times 5 \mathrm{~mm}$. Its diameter is around 7-8 mm leading to around 20 pixels in $\mathrm{x}$-y slice. The z-direction has a very large interval $(5 \mathrm{~mm})$ due to the need to reduce scanning time for patients. This results in only about 10-15 slices of this artery. Obviously this small amount cannot create a satisfied 3D wall at each time step. On the other hand, it is also not plausible to create slices between every pair of two neighbor slices by interpolating velocity fields of them, since the interpolation will integrate lots of noise vectors. Meanwhile, we know that the static TOF MRI images can reach high resolution (e.g., $0.5 \mathrm{~mm}$ ) in $\mathrm{x}, \mathrm{y}$, and $\mathrm{z}$ direction. It means that in $\mathrm{z}$ direction they can have around 10 extra slices between two neighbor slices of the 4D MRI data. Therefore we combines the two types of MRI images.

As shown in Fig. 2, accurate boundaries $\Theta$ over the high-resolution images are extracted which however are not dynamic. We then combine $\Lambda_{t}$ and $\Theta$ to generate high-resolution, dynamic pulsatile vessel walls. First, $\Lambda_{t}$ is used to compute a time-varying deformation factor $d_{t}$ of each slice (i.e., one location of the vessel). Second, $d_{t}$ is extended to high resolution by B-Spline interpolation at each high-resolution slice over each time step. Then the factor is used to morph $\Theta$ to reflect the dynamic change leading to an result contour. Finally, these contours are reconstructed to form the deforming vessel wall $\bar{\Theta}_{t}$ along $t$. These contours are also directly used to extract the final pulsatile flow from the velocity fields interpolated from $\vec{U}_{t}$.

\section{CASE STUDIES}

In the following case studies, 4D flow MRI images are scanned by a clinical 3T MRI system for neck area. The volume size of these velocity images is $392 \times 184 \times 10 \times 19 \times 3$. Here each two dimensional slice has a resolution of $392 \times 184$. There are only 10 slices exist in the vertical (z) direction (i.e., foot to head direction). Each slice indeed has 19 time steps representing one cardiac cycle. Here each pixel has 3 vector channels of blood flow velocity. The corresponding spatial resolution is $0.4427 \mathrm{~mm} \times 0.4427 \mathrm{~mm} \times 5 \mathrm{~mm}$ in physical space. The temporal resolution (interval between two time steps) is 50 milliseconds (ms). Because the carotid and vertebral arteries in the neck are oriented in a craniocaudal direction. The measurement range represented by parameters $V_{e n c}$ in $\mathrm{z}$ direction (from foot to head) is set to 1 meter/second and other two directions are 0.3 meter/second.

\subsection{Utilize Mean Flow Intensity to Extract Blood Flow Regions}

In segmentation implementation, there are two types of parameters. First, the Gaussian kennel variances in each direction used in Equation. 5 is set according to each direction's resolution. In the above case, the variance for $\mathrm{x}$, $\mathrm{y}$ direction is set to 2 , since the resolutions in these two directions are related high. The $\mathrm{z}$ direction variance is set as 0.5 due to the rather low resolution $(5 \mathrm{~mm})$ in this direction. Meanwhile, the variance for the time dimension is set to 1 . Second, the threshold used in segmentation from the mean flow intensity image is set to 0.6. It can be estimated according to the static features of the image. For instance, we can use a small area on an image corner (Figure 3(d)) to fit a normal distribution with mean value $\mu$ and variance $\sigma$, then this threshold is set to $\mu+2 \sigma$. In practice, we found a threshold form 0.5 to 0.65 is a good choice in our cases. These parameters 


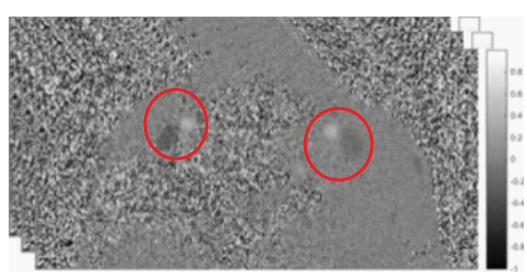

(a)

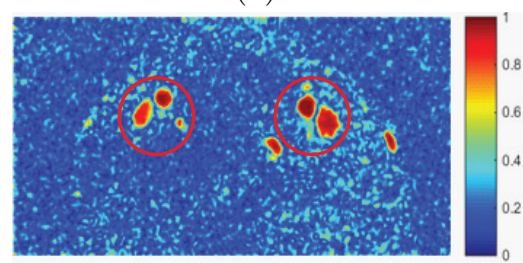

(d)

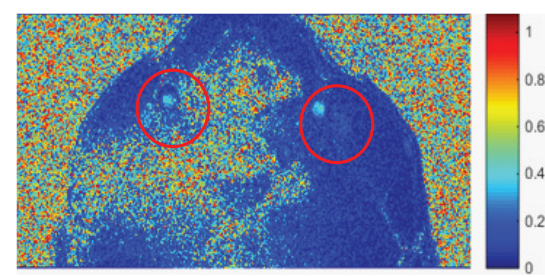

(b)

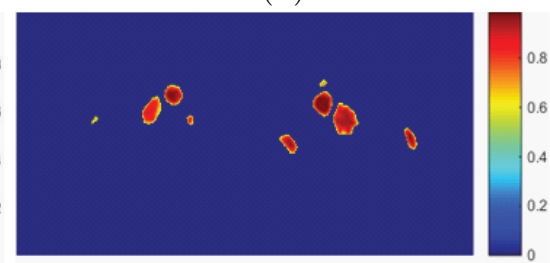

(e)

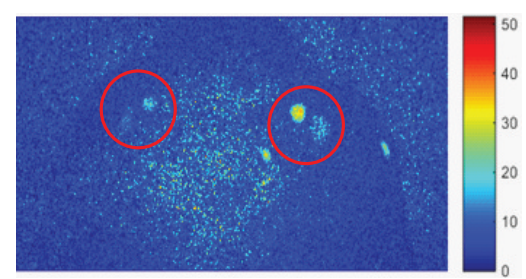

(c)

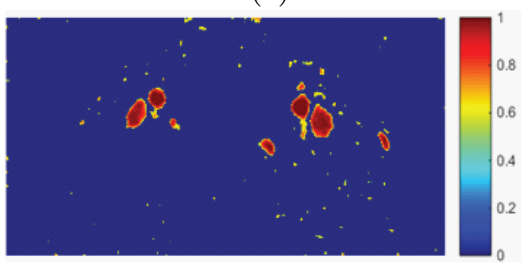

(f)

Figure 3. Using Mean flow intensity to extract blood flow field from the velocities on one slice scanned by $4 \mathrm{D}$ flow MRI on human neck. (a) shows 3 channel Velocity Images, (b) shows magnitude of velocity, (c) image feature used in reference, ${ }^{6}(\mathrm{~d})$ shows mean flow intensity map, (e)Segmentation result with threshold 0.6, (f) segmentation result without weighting method stated in 2.2. This fluid feature makes artery vessel regions very clear so that the vessel boundaries are easily found including carotid and vertebral arteries. In contrast, velocity magnitude image cannot distinguish vessel regions for these small arteries, as shown in the red circled locations.

can be adjusted after processing a small data set, then apply them to large data set automatically. In general, for the same MRI scanner with same scanning parameters, these segmentation parameters can be the same.

Figure 3(a) displays the velocity images of one slice at the first time step in a cardiac cycle. There are two coherent bright areas in the middle (inside the circles), which are the left and right carotid artery. The two coherent dark areas near them are two veins. Other vessels in this image are difficult to locate visually. Figure 3(b) shows the magnitude of velocity where some vessels like the two veins in circles is more difficult to identify. Figure 3(c) shows the image used in reference ${ }^{6}$ for segmentation, where the velocity magnitude times its corresponding MRI magnitude image to remove noise. The vessels in red circles are still fuzzy, especially in the left circle. In contrast, the mean flow intensity image shown in Figure 3(d) can locate the vessels easily. It is more robust to the magnitude changes. The carotid arteries and veins are manifested with high intensity values. Although they are very close to each other in space, they do not touch each other as the narrow but significant gap between them are distinguished, which yield rather good segmentation results with simple thresholding (0.5) in Figure 3(e). Figure 3(f) is the result without using the weighting method in section 2.2 to calculate mean flow intensity, where the quality if not good with more outliers.

\subsection{Extracting Blood Flow Regions During Diastole and Systole}

We test the robustness of the proposed method during diastole and systole. Figure 4(a) is the velocity magnitude in one carotid artery during diastole. At that time During systole, the mean velocity in carotid artery is the largest in the whole cardiac cycle. The small velocities (with blue color) make it very difficult to identify the vessel region. In contrast, Figure 4(b) shows the mean flow intensities where the vessel region (in yellow) can be easily recognized. Figure 4(c) shows vessel boundary is extracted by an active contour and overlays on Figure 4(a). Figure 4(d) shows the velocity magnitude during systole, where the velocities are larger (with yellow-orange colors). Figure 4(e) shows the mean flow intensities presenting a clear and enlarged region, and Figure 4(f) shows the extracted boundary. As showed in Figure 4, the proposed method is very robust to the dynamic change of velocity magnitudes in a cardiac cycle.

\subsection{Pulsatile Vessel Boundary and Blood Flow Visualization}

The 2D segmentation results are used together with TOF MRI images to create 3D pulsatile vessel and finally extract blood flow inside arteries as described in section 3. In this section, we show the extracted blood flow in 


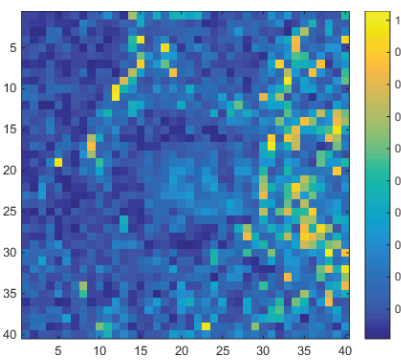

(a)

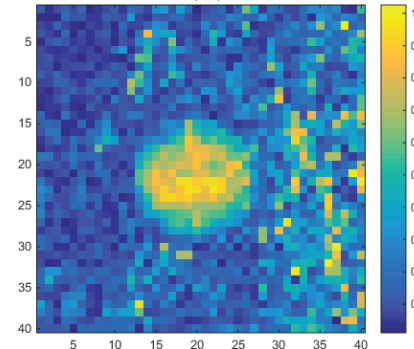

(d)

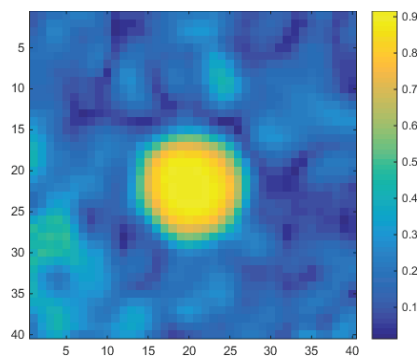

(b)

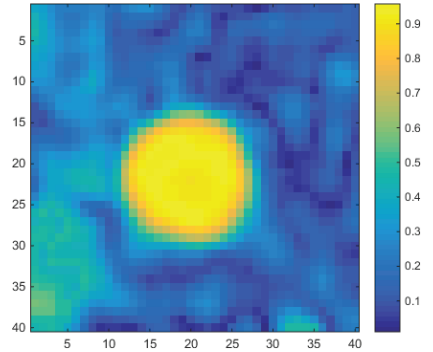

(e)

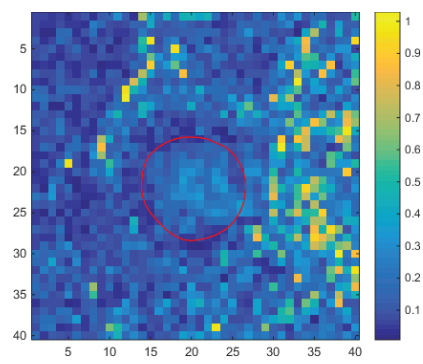

(c)

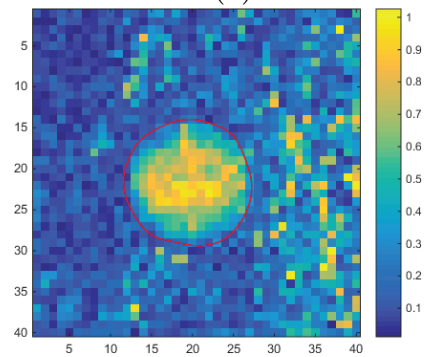

(f)

Figure 4. Blood flow field extraction during diastole $(\mathrm{a}-\mathrm{c})$ on first row and systole $(\mathrm{d}-\mathrm{f})$ on second row. the first column shows Velocity magnitude at that time, second column shows Mean flow intensity, third column shows extracted contour from mean flow intensity overlayed on the magnitude image.

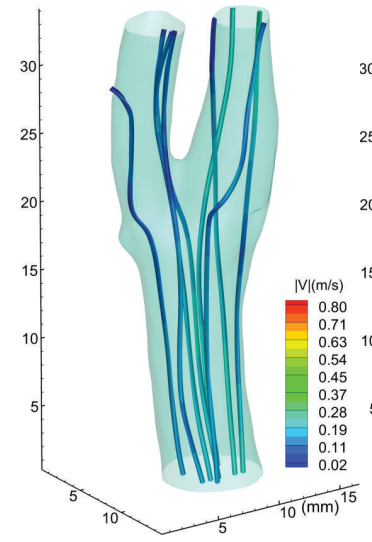

(a)

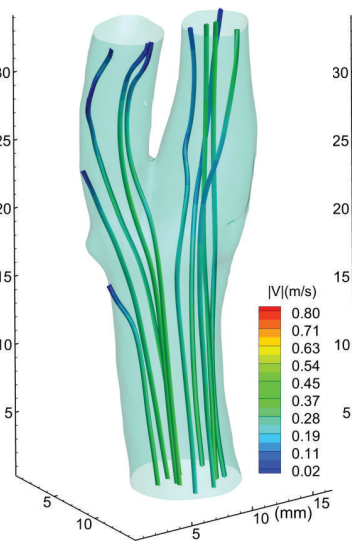

(b)

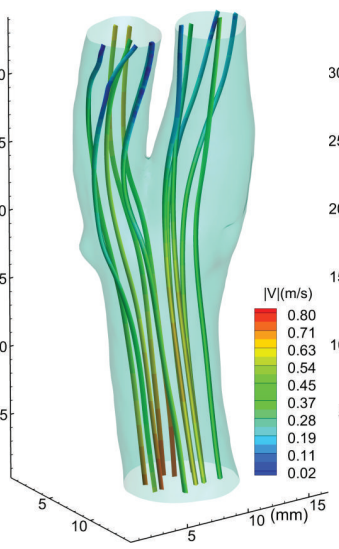

(c)

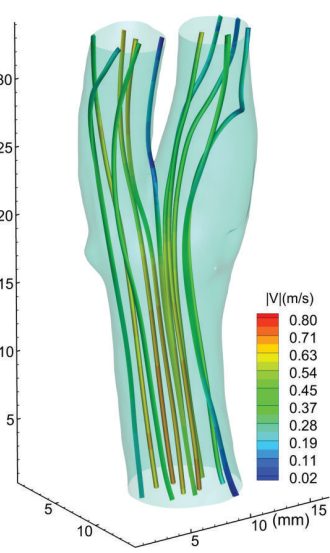

(d)

Figure 5. Pulsatile artery vessel and blood flow inside from diastole to systole with pulsatile artery boundary.(a) shows Velocity field during diastole, (b) shows the vessel 0.1 second after (a), (c)shows the vessel 0.15 second after (a), (d)shows the vessel 0.2 second after (a)

one carotid artery with pulsatile artery boundary. The static fine artery boundary is extracted from TOF MRI images with $0.3320 \mathrm{~mm} \times 0.3320 \mathrm{~mm} \times 1 \mathrm{~mm}$ spatial resolution.

The velocity field in a carotid with dynamic vessel wall shown in Figure 5, which demonstrates 4 frames related to 4 different times steps in a cardiac cycle from diastole to systole. Figure 5(a) shows the velocity field during diastole, when the flow rate is the smallest for artery inlet. At that time, the mean velocity speed in the carotid is low shown in blue streamlines. Then, heart systole begin, after 0.1 second, the velocity speed in artery begins to increase with high acceleration shown in green. Meanwhile, the size of the artery wall enlarges fast. After 0.15 second, flow rate is at peak, the mean velocity in artery is very large shown in yellow to orange. The boundary of the artery is even bigger in Figure 5(c). After 0.05 second, flow rate begin to decrease while the velocity in artery still very large but the size starts to slightly shrink. The artery movement can be seen on the video. We found this artery wall is very elastic. These features would indicate this carotid is from a healthy individual. 
Table 1. Segmentation quality evaluation for arteries.

\begin{tabular}{|l|l|l|l|l|}
\hline Arteries & level 1 & level 2 & level 3 & level 4 \\
\hline Carotid 1 & 16 & 2 & 1 & 0 \\
\hline Vertebral 1 & 15 & 2 & 2 & 0 \\
\hline Carotid 2 & 16 & 2 & 1 & 0 \\
\hline Vertebral 2 & 16 & 2 & 1 & 0 \\
\hline Carotid 3 & 15 & 2 & 2 & 0 \\
\hline Vertebral 3 & 15 & 3 & 1 & 0 \\
\hline
\end{tabular}

Table 2. Flow rate evaluation for 6 vessels

\begin{tabular}{|l|l|l|l|}
\hline Arteries & inlet $(\mathrm{ml})$ & outlet $(\mathrm{ml})$ & inlet / outlet \\
\hline Carotid 1 & 160 & 162 & 0.987 \\
\hline Vertebral 1 & 95 & 91 & 1.04 \\
\hline Carotid 2 & 184 & 180 & 1.02 \\
\hline Vertebral 2 & 88 & 85 & 1.04 \\
\hline Carotid 3 & 174 & 169 & 1.03 \\
\hline Vertebral 3 & 108 & 105 & 1.03 \\
\hline
\end{tabular}

\section{EVALUATION}

We performed the evaluation of our approach in two different ways. First, we evaluate the quality of our segmentation of vessel boundaries by radiologists. Second, we validate our extraction of 3D blood flow fields by evaluating the flow rate of mass in both qualitative and quantitative means.

\subsection{Segmentation Quality Evaluation}

The accuracy of the segmentation were evaluated by two experienced doctors. There were 3 carotid arteries and 3 vertebral arteries from three volunteers. For each volunteer, one carotid and vertebral artery was random selected. For each artery, one slice was selected for the velocity images in all time steps over a cardiac cycle. The segmentation results similar to Figure 4 were offered to the radiologists. The radiologists manually labelled the incorrect pixels which were included in the vessel region based their clinical knowledge and practice. Therefore, we could compute a ratio of the error pixels over the whole area of vessel. This ratio reflected the quality of our segmentation. We categorized the image segmentation quality into 4 levels. Level 1: excellent segmentation, no errors found; Level 2: good segmentation, the error ratio is smaller than $5 \%$; 3 . fair segmentation, the error ratio is between $5 \%$ and $10 \%$ error; Level 4: poor segmentation, the error ratio is larger than $10 \%$. Based on these rules, Table 1 reports the number of segmented images in each level, over the totally 19 images of each artery. It can be seen that most results (more than 80\%) are in Level 1, and more than $90 \%$ are on Level 1 and 2. Only 8 our of 114 are fair, while none is poor. This validation shows our segmentation method is rather successful.

\subsection{Flow Volume Rate Quantification}

The accuracy and internal consistency of the extracted 3D blood flow was validated based on the conservation of flow mass. This was demonstrated in Figure 6, where the blood flow rate is measured through a cardiac cycle at five locations spaced 10mm apart as shown in Figure 6(a). From the bottom to the top of the artery, there are 5 slices labeled as 1 to 5 . In Figure 6(b), for each slice, the flow rate (of the fluid mass) is plotted at each time step of one cardiac cycle to form a curved wave. The waves of flow rate in the first three slices have almost the same shape, since they are below the bifurcation located in the so-called Common Carotid Artery (CCA). The remainder two slices are above the artery bifurcation. We sum up the flow rate at both Internal Carotid Artery(ICA) and External Carotid Artery (ECA). The waves of these two slices are slightly different from the first three, while the area under each curve is almost the same (quantitative analysis will be showed later). This means that the extracted blood flow fields are without significate mass loss in one cardiac cycle. The slight shape difference between slices below and after bifurcation reflects different blood flow waveforms in CCA, ICA and ECA, which is affected by the artery bifurcation and elasticity $\left(\right.$ see $\left.^{17}\right)$.

We further conduct quantitative analysis. 3 carotid arteries and 3 vertebral arteries from 3 health volunteers are random selected. Then the velocity fields of all arteries are extracted for flow volume rate analysis. For all cases, the MRI scanning parameters and extraction parameters keep the same. For each artery, one inlet slice and one outlet slice, which are $3 \mathrm{~cm}$ away from each other, are selected. Each slice's flow rate of mass in the whole cardiac cycle is summed up, and the flow loss ratio between inlet and outlet is calculated. Table 2 shows the inlet flow rate and outlet flow rate, and their ratio respectively, of all 6 arteries. In one cardiac cycle, the flow loss ratio is below $5 \%$ for all cases. Vertebral arteries have smaller diameters compared to carotid arteries. They have a relatively higher flow lose $(3 \%$ to $4 \%)$ in one cycle. The reason may be because one incorrectly classified pixel during extraction would have more effect on the flow rate computation. In general, the loss ratio smaller than $5 \%$ shows very good quality of vessel segmentation and flow field extraction based on our method, since most existing methods have such a ratio bigger than $10 \%$. 

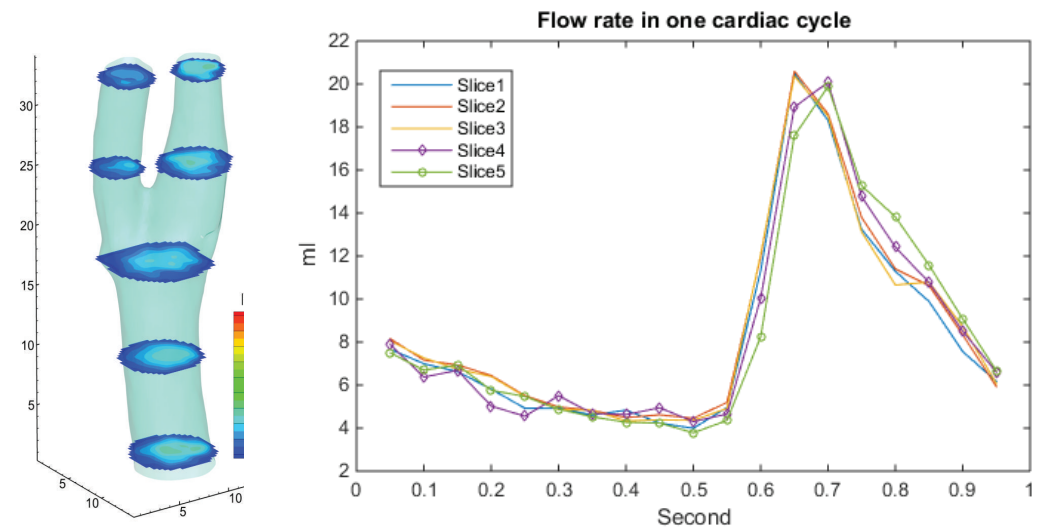

(a) Slice locations (b) Flow rate waves for 5 slices

Figure 6. Flow rate waves for 5 sampling slices on carotid artery during one cardiac cycle.

\section{CONCLUSION}

In this paper, we present new techniques to extract blood artery vessels from 4D flow MRI images, and then use them to rebuild pulsatile vessels in a cardiac cycle by deforming the accurate static vessel wall from TOF MRI images. Our work focuses on the segmentation based on a fluid feature, mean flow intensity, which provides robust, easy, and fast extraction from noisy and low-resolution 4D MRI data. The result deforming pulsatile vessel and dynamic blood velocity field will enable high-quality visualization and assessment in post-processing. Our next step work based on this paper is to (1) find more robust estimation method for this feature and combine multiple features to extract flow field; (2) visualize the dynamic blood flow with more advanced flow visualization techniques, according to physicians requirements; (3) use the pulsatile artery boundary as boundary condition for computational hemodynamics simulation. Then $4 \mathrm{D}$ flow MRI data can be further evaluated by comparing the measurement result with simulation results.

\section{ACKNOWLEDGMENTS}

This research was supported by Indiana University Health Value Fund and Kent State University Fellowship.

\section{REFERENCES}

[1] Stankovic, R., D.Allen, B., Garcia, J., Jarvis, K. B., and Markl, M., "4d flow imaging with mri," Cardiovascular Diagnosis and Therapy 4(2), 173-192 (2014).

[2] Hennemuth, A., Friman, O., C.Schumann, Bock, J., Drexi, J., and Huellebrand, M., "Fast interactive exploration of 4d mri flow data," SPIE Proceedings 7964, 1-12 (2011).

[3] Roldan-Alzate, A., Wahlin, A., Frydrychowicz, A., Niespodzany, E., Johnson, K., Wieben, O., and Reeder, S. B., "In vivo validation of $4 \mathrm{~d}$ flow mri for assessing the hemodynamics of portal hypertension," Journal of Magnetic Resonance Imaging 37(5), 1100-1108 (2013).

[4] Schrauben, E., Wahlin, A., Ambarki, K., Spaak, E., Malm, J., Wieben, O., and Eklund, A., "Fast 4d flow mri intracranial segmentation and quantification in tortuous arteries.," J Magn Reson Imaging. 42(5), 1458-1464 (2015).

[5] Markl, M., Frydrychowicz, A., Kozerke, S., Hope, M., and Wieben, O., "4d flow mri.," J Magn Reson Imaging 36, 10151036 (2012).

[6] Bustamante, M., Petersson, S., Eriksson, J., Alehagen, U., Dyverfeldt, P., Carlhall, C.-J., and Ebbers, T., "Atlas based analysis of 4d flow cmr: Automated vessel segmentation and flow quantification," Journal of Cardiovascular Magnetic Resonance 20(1), 181-187 (2015).

[7] Chan, T. F., Sandberg, B. Y., and Vese, L. A., "Vector filtering for color imaging," Journal of Visual Communication and Image Representation 11(2), 130-141 (2000). 
[8] Lukac, R., Smolka, B., Martin, K., Plantaniotis, K., and Venetsanopoulos, A., "Vector filtering for color imaging," IEEE Signal Processing Magazine 22(1), 74-386 (2005).

[9] Levin, A., Lischinski, D., and Weiss, Y., "A closed form solution to natural image matting," IEEE transactions on Pattern Analysis and Machine Intelligence 30(2), 228-242 (2008).

[10] Trip, R., Kuik, D., Westerweel, J., and Poelma, C., "An experimental study of transitional pulsatile pipe flow," Physics of Fluids 24(1), 014103:1-014103:17 (2012).

[11] Raffel, M., Willert, C., Wereley, S., and Kompenhans, J., [Particle Image Velocimetry: A Paractical Guide], Springer, second ed. (2007).

[12] Pope, S. B., [Turbulent Flows], Cambridge University Press (2000).

[13] P.Dyverfeldt, Gardhagen, R., Sigfridsson, A., Karlsson, M., and Ebbers, T., "On mri turbulence quantification," Magnetic Resonance Imaging 37(7), 913-922 (2009).

[14] Golshan, H., Hasaanzadeh, R., and Yousefzadeh, S., "An mri denoising method using image data redundancy and local snr estimation," Magnetic Resonance Imaging 31(7), 1206-1217 (2013).

[15] Kovesi, P., "Phase congruency detects corners and edges," 8th Digital Image Computing: Techniques an Applications 64, 136-148 (2003).

[16] Paris, S. and Durand, F., "A fast approximation of the bilateral filter using a signal processing approach," International Journal of Computer Vision 81(1), 24-52 (2009).

[17] N.Ku, D., "Blood flow in arteries," Fluid mechanics 29(1), 399-434 (1997). 